\title{
Fermeture annoncée et communication interne
}

\section{Marie-Pierre Strasbach et Marcel-Paul Cavallier}

\section{OpenEdition}

Journals

Édition électronique

URL : http://journals.openedition.org/communicationorganisation/2254

DOI : 10.4000/communicationorganisation.2254

ISSN : $1775-3546$

\section{Éditeur}

Presses universitaires de Bordeaux

\section{Édition imprimée}

Date de publication : 1 novembre 1999

ISSN : 1168-5549

\section{Référence électronique}

Marie-Pierre Strasbach et Marcel-Paul Cavallier, «Fermeture annoncée et communication interne », Communication et organisation [En ligne], 16 | 1999, mis en ligne le 27 mars 2012, consulté le 10 décembre 2020. URL : http://journals.openedition.org/communicationorganisation/2254; DOI : https://doi.org/10.4000/communicationorganisation.2254

Ce document a été généré automatiquement le 10 décembre 2020.

(c) Presses universitaires de Bordeaux 


\title{
Fermeture annoncée et communication interne
}

\author{
Marie-Pierre Strasbach et Marcel-Paul Cavallier
}

1 Un organisme voué à disparaître peut-il encore communiquer? Dans le cas d'une entreprise, la question pourrait sembler sans objet : alors que l'on voit bien pourquoi elle peut chercher à se faire reconnaître comme partenaire économiquement crédible, ou comme acteur institutionnel dans la cité ${ }^{1}$, on l'imagine moins se manifester si son activité cesse. Pour elle, il n'y a plus alors ni notoriété à établir, ni réputation à assurer, ni légitimité à justifier. Ainsi, à la cessation d'activité correspondrait fort logiquement une rupture de la communication : tant que l'on est en crise, c'est un enjeu de communiquer, mais cet enjeu n'est plus de mise lors du dénouement s'il est fatal. Les morts ne parlent pas.

2 La métaphore organique a ses limites : peu d'entreprises, actuellement moins que jamais, sont des individus isolés. Aux liens de dépendance (rapport d'établissement à son entreprise, filialisation, appartenance à un groupe industriel), s'ajoutent de multiples formes de partenariat et de réseaux qui solidarisent les entreprises entre elles ou avec d'autres acteurs socio-économiques ${ }^{2}$. Dès lors que les intérêts des anciens partenaires perdurent, un enjeu de pérennité demeure quels que soient les destins individuels. Aussi une entreprise peut-elle être amenée à mettre en scène sa propre disparition, surtout lorsque celle-ci n'est que la conséquence du désengagement partiel d'une entité plus vaste.

3 Le cas que nous exposons ici est révélateur de l'une de ces configurations. Il s'agit d'une entreprise - nous l'appellerons «les Houillères »- appartenant à un groupe industriel. Jusqu'à présent employeur principal d'un bassin, elle est confrontée à sa propre fermeture annoncée et programmée.

Dans le cadre d'un stage, nous avons participé aux activités en interne d'une cellule de communication décentralisée de l'entreprise. Il s'agissait de contribuer à l'élaboration d'un plan de communication qui, entre autres, exprimerait les choix stratégiques de l'entreprise quant à sa restructuration. Ce travail impliquait aussi d'être à l'écoute des 
salariés, de leurs déceptions, de leurs récriminations, de leurs attentes. C'est dans ces perspectives (quel message émettre, comment est-il reçu?) que nous avons recueilli les données qui fondent cette étude.

5 En suivant le fil du travail élaboré au sein de la cellule de communication, on peut bien sûr aborder la question instrumentale telle qu'elle se pose du point de vue de l'entreprise : comment préparer les partenaires externes et internes au processus de son retrait? Ce faisant, on risque cependant de négliger les aspects relationnels et identitaires - particulièrement forts dans la situation présente - qui se jouent dans toute communication. Aussi tenterons-nous dans un second temps de privilégier ces aspects, en nous appuyant principalement sur ce que construisent les acteurs eux-mêmes: l'information, qu'ils se réapproprient ; mais plus encore, leur propre identité.

\section{La communication comme problème}

\section{Un objectif instrumental : la nécessité d'informer}

6 La relation de communication interne avec le personnel s'inscrit dans un ensemble plus global, celui d'une politique des ressources humaines. Pour les Houillères, cette politique se focalise désormais sur le problème essentiel de l'avenir de ses salariés. Dans ce cadre, plusieurs types de missions devraient se faire jour pour préparer le processus de retrait de l'entreprise :

7 - une mission de consensus, cherchant à éviter l'explosion sociale et les manifestations d'opposition au processus en cours ;

8 - une mission de maintien, malgré les événements annoncés, d'un minimum de dynamisme et de motivation des salariés, afin que l'entreprise soit à même de poursuivre dans les meilleures conditions ses activités d'ici la fermeture;

9 - une mission d'information: il s'agit d'aider les salariés à préparer leur avenir, en les informant le plus loyalement possible sur leur sort, et sur les alternatives qui s'ouvrent à eux. C'est principalement cette troisième mission qui entre dans les attributions de la communication; mais celle-ci ne peut aborder cette mission indépendamment des deux autres.

\section{Des messages contradictoires}

10 L'avenir des salariés des Houillères est régi par deux documents contractuels :

11 - un protocole de conversion (26 janvier 1989), qui envisage pour ceux qui le souhaitent, les modalités de leur départ hors du groupe, assorties de possibilités de retour ;

12 - un « Pacte charbonnier » (20 octobre 1994) qui a pour objet la poursuite de l'activité de l'entreprise jusqu'en 2005 dans la paix sociale, et qui garantit l'emploi même au-delà de cet horizon.

13 Dans ce contexte, le salarié est placé devant deux possibilités de projets, qui sont de nature opposée :

14 - l'accès à un emploi ou le développement d'une activité hors de l'entreprise ; 

peuvent être vus comme complémentaires, plutôt que contradictoires. Les salariés se contentent d'y voir une alternative, dans laquelle ils trouveront un éventail de compensations aux désavantages liés à la perte future de l'activité; or, plus ces compensations sont variées, plus s'accroît leur liberté de choix, et plus ils augmentent les chances d'y trouver leur compte.

\section{Un message ambigu et peu crédible}

21 La liberté de choix qui est laissée au destinataire repose sur l'ambiguïté des messages. En contrepartie, l'émetteur, qui doit assumer cette ambiguïté, est en porte-à-faux. Cette position inconfortable, il la doit au côté contourné du message. Ainsi, le mot " fermeture ", considéré comme trop brutal, est banni. À cette approche toute en nuances et en euphémismes sur des équivalents sémantiques de la fermeture, les mineurs préfèrent s'en tenir à des évidences simples, telles que «fermeture = chômage »: «je pense qu'il vaut mieux payer 15000 mineurs que 15000 chômeurs $»^{3}$. 
22 En outre, celui qui doit assumer la position d'émetteur est déstabilisé du fait des intentions mêmes du message. En effet, ce qu'il tente de faire accomplir est fondamentalement difficile à admettre.

Il s'agit d'aboutir à la cessation des activités de l'entreprise. D'un point de vue moral, cet objectif est difficilement acceptable par des salariés pour qui cette activité se confondait avec leur vie. D'un point de vue économique, cet objectif, concevable pour une entreprise en quête de rentabilité, est paradoxal pour des salariés qui jugeaient de la pertinence de leur activité selon des critères de service public, faisant plus de place aux besoins nationaux d'énergie qu'à la rentabilité (on pourrait facilement greffer là-dessus des propos sur la "casse industrielle ", quoique la sociologie politique locale ne s'y prête guère).

Ce qui est difficile à admettre, c'est la présentation sous forme positive d'une nouvelle qui ne peut pas être appréhendée comme telle. D’habitude, la communication descendante «positive» les messages diffusés, ce qui se fait le plus souvent en se limitant à des informations de travail, ou à une sélection de bonnes plutôt que de mauvaises nouvelles. Or, ce genre de pratique convient peu à une nouvelle comme la cessation d'activité, à propos de laquelle on ne peut guère transmettre d'aspects positifs. On rejoint ici un problème classique en communication de crise : comment informer pour prévenir d'un danger qui menace, tout en rassurant? La communication doit alors concilier deux rôles, fort peu conciliables :

26 - un rôle traditionnel de diffuseur d'informations destinées à contribuer à la bonne marche de l'entreprise et à la valoriser; pour l'occasion, ce rôle se transforme en celui d'un diffuseur d'euphémismes du genre "pas d'affolement, la situation n'est pas si grave »;

27 - un rôle nouveau pour elle, et d'autant plus innovant, et déstabilisant, qu'il prend ce rôle traditionnel à contre-pied : faire connaître les conséquences de la crise ou de la récession que subit l'entreprise.

\section{Un émetteur déstabilisé}

28 Le communicant, qui est considéré comme l'émetteur du message, n'en est pas le responsable ${ }^{4}$, mais doit assumer les propos de l'entité qui l'emploie, les Houillères. Or son propre statut est en cause, car l'entité doit disparaître, et le communicant cessera par conséquent d'y appartenir. Il est amené à parler au nom de quelque chose dont il doit luimême se déprendre. Pire encore, il doit argumenter sur les formes de pérennité de l'emploi, et rassurer ses collègues sur leur avenir, alors qu'il est lui-même amené à douter du sien. Si son reclassement est acquis, il ne l'est pas nécessairement dans l'activité de communicant. En outre, dès lors que ce ne sont plus les Houillères mais une nouvelle entité, les Charbonnages de France, qui garantit l'emploi, le communicant doit mettre en avant cette nouvelle entité qui est une abstraction aussi bien pour les mineurs que pour lui-même.

\section{Une identité en question}

29 Les destinataires, quant à eux, se montrent peu réceptifs aux propositions qui leur sont faites. Alors qu'on leur offre une possibilité de sortie avantageuse, ou mieux encore, des 
garanties de statu quo, ils sont méfiants ou traumatisés, et leur incompréhension semble totale. identitaire. L'attachement des salariés à leur travail actuel est fort. D'ordinaire, un tel mais, dans la situation actuelle de crise, c'est précisément cette identité que l'entreprise tente vainement de déconstruire.

\section{La communication comme opportunité}

35 En adoptant une approche instrumentale, nous avons jusqu'ici envisagé la communication comme un moyen subordonné aux objectifs de l'entreprise. Celle-ci 
cherche à mettre en œuvre un changement planifié (sa fermeture programmée), et la communication est un moyen utilisé pour le faire accepter. des pratiques de communication essentiellement directives: "La mine c'est comme l'armée. Tu le fais et tu te tais ». Ce qui était appelé « participation » servait plutôt, dans le quotidien de l'entreprise, à faire entériner des décisions déjà prêtes. La marge de négociation était faible, limitée à des questions secondaires. Houillères était d'autant mieux acceptée que l'entreprise était beaucoup plus qu'un simple employeur, elle était l'employeur local de référence ; très fortement investie dans la vie locale et dans les équipements collectifs, elle y assumait un rôle institutionnel fort. Ainsi, les salariés se sentaient tenus par un employeur auquel ils devaient beaucoup, et dont dépendaient aussi bien leur vie professionnelle que leur vie de tous les jours, au village ou à la ville. Les Houillères assuraient non seulement l'intégration systémique, en tant qu'entreprise, mais aussi l'intégration sociale et normative de ses salariés, en tant qu'institution $^{11}$; et elles jouaient pleinement ce rôle. 

Houillères, la sidérurgie : «Tout le monde dans la maison pensait que la crise, c'était quelque chose qui était dehors, une bête maléfique qui un beau jour allait disparaître. [...] Le premier message a été de dire : la crise n'existe pas. Nous sommes un organisme vivant et, si nous n'avons pas la capacité d'adaptation, c'est notre problème. Donc la crise, c'est notre crise à nous, les hommes, l'entreprise ; c'est la crise de nos rigidités et de l'incapacité à nous adapter. La réponse que l'on doit apporter à cela, c'est la réactivité14 "Suite à ce diagnostic, le dircom de Sollac élabore un programme de "réactivité ", qu'il s'agit de communiquer à l'entreprise dans son ensemble. Certes, cette approche volontariste se situe dans une perspective de continuation et de pérennité de l'activité originelle de son groupe, ce qui n'est plus le cas pour les Houillères, il n'empêche : il s'agit bien, dans un cas comme dans l'autre, de prendre à contre-pied des situations acquises, situations suffisamment ancrées socialement pour que seule une catastrophe puisse les remettre en cause.

\section{Des opportunités de mobilisation}

Si la fermeture des Houillères crée des opportunités, celles-ci ne se limitent pas à la seule communication, ni au seul cadre des Houillères. Ainsi, dans le cadre du groupe Charbonnages de France, on peut voir dans le choc créé par la restructuration un argument pour rationaliser les activités, en se défaisant de ce qui est insuffisamment 
rentable. Ou encore, dans le cadre du bassin d'emploi, la restructuration est un argument pour accueillir des activités nouvelles, porteuses de meilleures perspectives d'avenir : les réticences traditionnelles vis-à-vis de ce qui est étranger à l'activité minière n'ont plus de raison d'être, parce qu'il n'y a plus le choix ${ }^{15}$.

À l'intérieur même des Houillères, la restructuration crée aussi des opportunités dès lors qu'elle remet en cause des habitudes acquises.

51 Toutes habitudes de travail collectif, tout monde social, font émerger un ensemble d'usages, de procédures, d'habitudes, de routines, de règles, qui sont à la fois des raccourcis cognitifs (ils permettent d'éviter de se poser des problèmes à résoudre lors de chacune de nos décisions), et des garanties sociales : on peut s'attendre à ce qu'autrui ne s'écarte pas trop de ces pratiques, et si on ne s'en écarte pas trop soi-même, les uns et les autres devraient arriver à se comprendre, et donc à coopérer, et l'ensemble devrait fonctionner de façon à peu près satisfaisante. Tous ces dispositifs peuvent être renforcés par des signes tangibles d'engagement (de gros investissements par exemple), qui prouvent à autrui que la situation est destinée à durer. On peut alors travailler en confiance, dans un cadre à peu près fixe. C'est ce genre de situation qu'ont établie les Houillères, la garantissant par des investissements industriels (comme toute grande entreprise l'aurait fait), mais aussi par une présence forte et bénéfique au sein de la communauté locale.

52 L'inconvénient de ces mécanismes découle de leur avantage même: à trop vouloir garantir les situations, à trop vouloir donner des gages, ils figent les activités, voire les ritualisent. Or, dans les situations quotidiennes de travail, il y a peu de raisons de dénoncer ce qui peut devenir une sclérose. Seules les situations de changement (et la restructuration en est un ideal-type) sont à même de fournir un argumentaire qui permette de débloquer la situation. D'où le rôle clef, en ces circonstances, de la communication.

53 Mais une autre raison concourt à ce rôle clef : en effet, la restructuration peut être caractérisée comme une situation où disparaissent les gros investissements, « objets » qui garantissent un engagement de l'entreprise. Ces objets disparaissant, il faut bien disposer de mots pour compenser leur absence, et pour réaffirmer un engagement qui n'est plus suffisamment étayé sur le plan matériel.

54 Ainsi, non seulement la crise fournit un argumentaire, mais elle rend la communication plus nécessaire encore. La crise en stimule à la fois l'offre (l'argumentaire : « il y a quelque chose à dire »), et la demande (la nécessité de dire quelque chose, face à un public qui autrement serait privé de tout signe en provenance de l'entreprise).

\section{Quelles références?}

Les Houillères, qui n'ont pas établi jusqu'à présent de courant de communication de ce type, peuvent-elles devenir du jour au lendemain un émetteur légitime de messages affirmant un engagement, alors qu'elles accumulent les signes contraires? Elles s'étaient jusqu'à présent donné d'autres moyens de convaincre, mais ces moyens étaient matériels, et non symboliques. L'entreprise pouvait se passer de mots, dès lors qu'elle investissait, produisait et fournissait des emplois. Comment à la fois changer de mode d'expression, et faire passer l'idée de changement? et ce, en introduisant de surcroît des nuances dialectiques quant à la continuité, à la pérennité de l'activité ? 

préalablement à la restructuration, une fonction phatique. Et qu'ainsi, les flux de communication nécessités par la situation actuelle de restructuration s'inscrivent dans la continuité de ce qui se faisait auparavant. Sinon, comment argumenter sur la pérennité ?

d'un message portant sur la pérennité d'une activité - qu'elle se maintienne dans l'entreprise ou en dehors de celle-ci - devrait pouvoir décrire l'actuel changement ( i.e. la restructuration et ses implications) en termes d'action programmée, plutôt qu'en termes de crise. Cela signifie: présenter la fermeture comme l'étape d'un processus maîtrisé, et non comme un événement fatal, terminal et incontrôlé. Pouvoir tenir ce type de discours implique des choix stratégiques, mais aussi tout un travail de préparation. En temps de crise, la communication de l'entreprise est d'abord jugée d'après l'image qu'elle s'est forgé en temps normal. D'où l'importance de forger en période calme une véritable politique de communication.

Or, c'est plutôt la crainte de conflits éventuels provoqués par la perspective de fermeture qui a motivé la mise en place par les Houillères d'un dispositif de communication de crise. C'est seulement lors de la restructuration que la démarche de communication, du moins dans sa dimension reclassement, a été inaugurée. Comment cette démarche pourrait-elle être légitime, alors que la direction s'en passait volontiers avant cette crise ? Privée d'un passé qui la fonde, elle est aussi privée d'un avenir qui la justifierait : « la communication se construit dans la durée, or que veut dire la durée quand une unité va fermer ? ${ }^{16}$.

\section{L'offre d'informations émanant de la presse locale} possibilité de se tourner vers des sources concurrentes, et elles ne manquent pas.

Concurrence en provenance de l'extérieur tout d'abord, avec la presse locale : celle-ci est lue assidûment par les salariés, et peut même être considérée comme une sorte de second journal interne. Toutes les informations concernant les Houillères y sont d'autant plus crédibles qu'elles sont relatées par un journaliste.

61 Or, alors qu'un service communication saura diriger les informations en y sélectionnant ce qui va dans le sens de la politique poursuivie par l'entreprise (ici : la fermeture), de nombreux articles de presse se situent dans un registre différent, voire contraire. Ainsi, sont mis en avant des thèmes tels que la modernisation des Houillères, l'amélioration de la productivité : « cette surproduction servira à compenser les pertes éventuelles d'autres unités $»^{17}$. Dans cet exemple, la presse locale souligne la qualité des performances d'un site, alors même que celui-ci est destiné à fermer. On est loin des arguments développés par les Houillères, qui soulignent plutôt la faible rentabilité de la mine et la compétitivité de la concurrence étrangère.

62 Sans doute peut-on voir dans cette attitude de la presse un déphasage plutôt qu'une intention de contrer la politique des Houillères. Dans ce bassin d'emploi, la presse a longtemps été une sorte relais institutionnel, et s'est habituée à valoriser assez systématiquement les initiatives de l'employeur dominant de ce bassin, et ce d'autant plus qu'il contribuait puissamment au développement local.

63 Tel n'est plus le cas, même si les Houillères préparent leur succession en cherchant à induire localement d'autres emplois. Mais cette politique, entièrement nouvelle par rapport aux mentalités, ne pourra être comprise qu'avec un certain retard par les 
habitants; et la presse locale, qui a pour vocation d'informer plutôt que de former, peut très bien se contenter de refléter ces mentalités et de les conforter.

\section{L'offre d'informations informelles} sont surtout les circuits informels qui risquent de supplanter sa démarche de communication. Ainsi, une multitude d'informations sur la restructuration proviennent de salariés pris individuellement ou collectivement, ou de leurs représentants institutionnels, les syndicats. C'est un sentiment général qu'exprime ce responsable d'une cellule de communication: "les partenaires sociaux sont plus rapides que les informations officielles; notre rôle est d'essayer de les devancer ». Une étude spécifique mériterait d'être consacrée aux partenaires sociaux et à leur rôle en interférence avec les informations officielles. l'avenir de chacun est bienvenue, et plus encore si elle parvient par un canal informel. En effet, ce qui émane des Houillères, qui ont déçu pour de multiples raisons (en perte de légitimité, porteuses de mauvaises nouvelles, destructrices des références), est considéré avec méfiance. On accueillera beaucoup plus volontiers ce qui parvient par d'autres canaux.

Ainsi, celui qui, transmettant des nouvelles, rompt un secret ${ }^{18}$, apparaît alors comme le détenteur d'un savoir précieux. En outre, en montrant qu'il fréquente des sources bien informées, il donne une preuve de ce que ces sources sont accessibles. C'est un motif d'espoir : malgré les événements, on n'est plus tout à fait le jouet des mesures qui vous échappent. Bref, les informations informelles font du bien à ceux qui les énoncent, et aussi à ceux qui les écoutent.

\section{Une identité sociale retrouvée?}

Face à la perte de sens qui menace les salariés, les circuits d'information informelle semblent offrir une forme de réponse plus satisfaisante que celle des canaux formels. La communication informelle soulage, elle permet un écoulement socialement acceptable de l'agressivité refoulée, elle sert d'exutoire en période de crise.

Plus encore qu'au plan individuel, c'est sur le plan de la vie du groupe que la communication informelle est efficace. Elle est mobilisée pour défendre la cause du groupe menacé dans son intégrité par les mesures de mobilité : ainsi court la rumeur que d'autres mines (où certains membres du groupe doivent être mutés, à la suite d'autres qui l'ont déjà été) respectent moins bien les consignes de sécurité (ce qui est étayé par un accident dont a été victime un ancien membre du groupe, muté dans une autre mine).

Enfin et surtout, la communication informelle est un véhicule efficace de cohésion sociale. En effet, c'est dans des discussions collectives qu'émergent des opinions que tous partagent, ce qui donne consistance au groupe.

En des circonstances menaçantes pour ses membres, l'intégration du groupe passe par une solidarité unanime face au danger; la divergence d'opinion serait plus qu'une rupture, elle s'apparenterait à une trahison: ce qui justifie plus que jamais le côté fusionnel du monde de la mine, où seul le consensus est toléré19.

Communication et organisation, 16 | 2012 


\section{Conclusion}

71 informer des salariés qui subissent les effets d'une restructuration. Nous avons constaté les difficultés de la démarche: en effet, tant qu'il s'agit d'informer les salariés d'un constat, on se trouve dans une sorte d'impasse, celle de la communication piégée par une information descendante. Et, lorsque ce constat est celui d'un échec (ou d'une fermeture annoncée), on ne voit guère ce qu'ils auraient à en attendre ou à en retirer.

Or, précisément parce qu'il y a crise, le changement induit par celle-ci suscite la remise en cause de cette vision statique et unidirectionnelle. La crise redistribue les cartes, elle est porteuse de nouvelles opportunités de mobilisation et de communication; elle met en avant de nouveaux acteurs. Peut alors se développer une communication qui permette à chacun des salariés de donner un sens à sa vie professionnelle, de se reconnaître dans un groupe, et qui permette au groupe d'avoir une vie qui lui soit propre : enjeu essentiel dans des circonstances où l'individu, ou le groupe, se sentent menacés dans leur identité même.

\section{NOTES}

1. Ce thème donne lieu à un grand nombre de publications, parmi lesquelles il faut mentionner : Saisaulieu Renaud, L'entreprise, une affaire de société, Paris, Presses de la Fondation Nationale des Sciences Politiques, 1990 ; Le Goff Jean-Pierre, Le mythe de l'entreprise, Paris, La Découverte, 1993 ; d'Almeida Nicole, L'entreprise à responsabilité illimitée, Paris, Éd. Liaisons, 1996.

2. Paché Gilles, Paraponaris Claude, L'entreprise en réseau, Paris, PUF, 1993.

3. Propos recueilli par Bur Cathy, Rapport de stage de DEA, Université de Paris VII, 1995.

4. Cf. Goffman Erwin, Les cadres de l'expérience, Paris, Éd. Minuit, 1991.

5. Cf. Coch Lester, French John, « Overcoming resistance to change », Human Relations, I (1948).

6. Propos recueillis par Strasbach Marie-Pierre, Fermeture annoncée, pacte charbonnier et communication interne, Mémoire de DESS, Université de Nancy 2,1996.

7. Cf. Francfort, Osty, Sainsaulieu, Uhalde, Les mondes sociaux de l'entreprise, Paris, Desclée de Brouwer, 1995.

8. Strasbach Marie-Pierre, ibid.

9. Propos recueillis par Strasbach Marie-Pierre, ibid.

10. On parle alors de changement émergent, c'est-à-dire d'un changement dont le sens émerge des conversations entre les acteurs et des interprétations qu'ils en font. Sur l'opposition entre les deux notions de changement, cf. Taylor James, «La dynamique de changement organisationnel. Une théorie conversation/texte de la communication et ses implications ", et Demers Christiane, «La diffusion d'un changement radical: un processus de redéfinition et de restructuration de l'organisation ", Communication \& Organisation n 3, mai 1993.

11. Cf. Quéré Louis, "Communication sociale: les effets d'un changement de paradigme", Réseaux $n^{\circ}$ 34, mars 1989.

12. Cf. Balle Francis, Médias et sociétés, Paris, Éd. Montchrestien, 1980. 
13. Cf. Hermès $n^{\circ} 11-12$ «À la recherche du public », 1993.

14. Bertrand Alain, "La communication stratégique. L'exemple de Sollac », in Paul-Cavallier Marcel, Vingt ans de communication d'entreprise en Lorraine, Nancy, PUN, 1992.

15. Mirgaine Anne-Marie, Olszak Alfred, in Paul-Cavallier Marcel, ibid.

16. Propos recueilli par Strasbach Marie-Pierre, ibid.

17. Article paru en août 1996.

18. Petitat André, Secret et formes sociales, Paris, PUF, 1998.

19. Sainsaulieu Renaud, L'identité au travail, Paris, Presses de la Fondation Nationale des Sciences Politiques, 1977.

\section{RÉSUMÉS}

La restructuration d'une entreprise minière amène celle-ci à programmer la fermeture de ses unités d'exploitation, tout en garantissant le réemploi de ses salariés. La communication interne de l'une de ces unités, étudiée ici, soulève de multiples difficultés. Les communicants, de par la situation de crise, risquent d'avoir à émettre des messages contradictoires. Face à la crise, les salariés se sentent menacés dans leur identité même, plus encore que dans leur avenir. Comment, dans ces conditions, une entreprise peut-elle définir une démarche de communication interne qui soit en phase avec son personnel?

Restructuring of a mining company leads to programming the closing down of its plants, while guaranteeing re-employment of its workers. Inner communication of one of these plants, here studied, is in trouble. Due to the crisis, the transmitters may have to express contradictory messages. Facing this crisis, the workers feel threatened in their own identity, even more than by their future. Therefore, how can the firm define a process of inner communication better adapted to its staff?

\section{AUTEURS}

\section{MARIE-PIERRE STRASBACH}

Marie-Pierre Strasbach, titulaire d'un DESS communication des entreprises et des organisations, est intervenante en communication à EDF.

\section{MARCEL-PAUL CAVALLIER}

Marcel Paul-Cavallier est Maître de Conférences en sciences de l'information à l' Université de Nancy 2, et membre du Groupe de Recherche Information, Communication et Propagandes. 